\title{
Predictors of sinonasal quality of life and nasal morbidity after fully endoscopic transsphenoidal surgery
}

\author{
Andrew S. Little, MD, ${ }^{1}$ Daniel Kelly, MD, ${ }^{2}$ John Milligan, MD,, Chester Griffiths, MD, ${ }^{2}$ \\ Daniel M. Prevedello, MD, ${ }^{3}$ Ricardo L. Carrau, MD, ${ }^{3}$ Gail Rosseau, MD, ${ }^{4}$ Garni Barkhoudarian, MD, ${ }^{2}$ \\ Bradley A. Otto, MD, ${ }^{3}$ Heidi Jahnke, RN, MSN, ${ }^{1}$ Charlene Chaloner, RN, ${ }^{2}$ Kathryn L. Jelinek, RN, ${ }^{3}$ \\ Kristina Chapple, PhD, ${ }^{1}$ and William L. White, MD'

\begin{abstract}
1Division of Neurological Surgery, Barrow Neurological Institute, St. Joseph's Hospital and Medical Center, Phoenix, Arizona; ${ }^{2}$ Brain Tumor Center \& Pituitary Disorders Program, John Wayne Cancer Institute, Santa Monica, California; ${ }^{3}$ Department of Neurosurgery, The Ohio State University, Columbus, Ohio; and ${ }^{4}$ Department of Neurosurgery, NorthShore University HealthSystem, Evanston, Illinois
\end{abstract}

OBJECT Despite the increasing application of endoscopic transsphenoidal surgery for pituitary lesions, the prognostic factors that are associated with sinonasal quality of life (QOL) and nasal morbidity are not well understood. The authors examine the predictors of sinonasal QOL and nasal morbidity in patients undergoing fully endoscopic transsphenoidal surgery.

METHODS An exploratory post hoc analysis was conducted of patients who underwent endoscopic pituitary surgery and were enrolled in a prospective multicenter QOL study. End points of the study included patient-reported sinonasal QOL and objective nasal endoscopy findings. Multivariate models were developed to determine the patient and surgical factors that correlated with QOL at 2 weeks through 6 months after surgery.

RESULTS This study is a retrospective review of a subgroup of patients studied in the clinical trial "Rhinological Outcomes in Endonasal Pituitary Surgery" (clinical trial no. NCT01504399, clinicaltrials.gov). Data from 100 patients who underwent fully endoscopic transsphenoidal surgery were included. Predictors of a lower postoperative sinonasal QOL at 2 weeks were use of nasal splints $(p=0.039)$ and female sex at the trend level $(p=0.061)$; at 3 months, predictors of lower QOL were the presence of sinusitis $(p=0.025)$, advancing age $(p=0.044)$, and use of absorbable nasal packing ( $p$ $=0.014$ ). Health status (multidimensional QOL) was also predictive at 2 weeks $(p=0.001)$ and 3 months $(p<0.001)$ and was the only significant predictor of sinonasal QOL at 6 months $(p<0.001)$. A Kaplan-Meier analysis was performed to study time to resolution of nasal crusting, mucopurulence, and synechia as observed during nasal endoscopy after surgery. The mean time $( \pm$ SEM) to absence of nasal crusting was $16.3 \pm 2.1$ weeks, mucopurulence was $6.2 \pm 1.1$ weeks, and synechia was $4.4 \pm 0.5$ weeks. Use of absorbable nasal packing was associated with more severe mucopurulence.

CONCLUSIONS Sinonasal QOL following endoscopic pituitary surgery reaches a nadir at 2 weeks and recovers by 3 months postoperatively. Use of absorbable packing and nasal splints, while used in a minority of patients, negatively correlates with early sinonasal QOL. Sinonasal QOL and overall health status are well correlated in the postoperative period, suggesting the important influence of sinonasal QOL on the patient experience.

http://thejns.org/doi/abs/10.3171/2014.10.JNS141624

KEY WORDS quality of life; endoscopic surgery; transsphenoidal surgery; pituitary adenoma; sinusitis; pituitary surgery

$\mathrm{T}$ RansSPhenoIDal surgery is the treatment of choice for most patients with symptomatic pituitary lesions. Because transsphenoidal surgery exploits the nasal corridor and paranasal sinuses to reach the skull base, sinonasal trauma is a primary source of postoperative morbidity in many of these patients. Reported rhinological complications from transsphenoidal surgery include sinusitis, synechia, anosmia, epistaxis, septal perforation, nasal congestion, and impaired mucociliary clearance. ${ }^{1,4,5,8,18}$ This morbidity can impact nasal functioning, patient's satisfaction with surgery, and quality of life (QOL)., ${ }^{2,3,6,13,16}$ Fully endoscopic transsphenoidal surgery has been adopted by

ABBREVIATIONS ASK Nasal-12 = Anterior Skull Base Nasal Inventory-12; QOL = quality of life; SF-8 = 8-item Short-Form Health Survey. SUBMITTED July 21, 2014. ACCEPTED October 16, 2014.

INCLUDE WHEN CITING Published online April 3, 2015; DOI: 10.3171/2014.10.JNS141624.

DISCLOSURE Dr. Little is an investor in Kogent Surgical. Dr. Kelly receives royalties from Mizuho. Barrow Neurological Foundation provided funding for the study. 
many pituitary surgical teams because it affords superior panoramic visualization of the operative field compared with microscopic techniques. ${ }^{7,9,14}$ A better understanding of the surgical and patient factors that influence postoperative sinonasal QOL and nasal morbidity may be helpful for improving patient counseling and surgical technique. In this study, we describe an exploratory post hoc analysis of a multicenter QOL study to define predictors of sinonasal morbidity and QOL after endoscopic transsphenoidal surgery.

\section{Methods \\ Study Design}

A post hoc analysis was conducted using data compiled during a prospective multicenter cohort study ("Rhinological Outcomes in Endonasal Pituitary Surgery" [clinical trial no. NCT01504399, clinicaltrials.gov]) of 2 commonly used transsphenoidal surgery techniques (a direct uninostril microscopic endonasal approach and a fully endoscopic endonasal approach). The study was approved by the institutional review boards at the 4 participating institutions. Informed consent was obtained from all study participants. The study adhered to the principles set forth in the US Code of Federal Regulations, Title 45, Part 46, Protection of Human Subjects, Revised January 15, 2009.

\section{Patient Population}

Eligible patients (consecutive English- or Spanishspeaking adults [18 years or older] with sellar pathology who had a planned primary or secondary transsphenoidal surgery) were identified in the outpatient neurosurgical clinics at 4 US pituitary centers between October 2011 and August 2013. Patients with a history of prior radiotherapy to the paranasal sinus region or skull base were excluded. A total of 301 patients were screened for eligibility; 27 patients were not eligible for the study and 39 declined to participate. From the remaining population, 123 patients received fully endoscopic surgery under the protocol. The surgeries were performed by 3 teams at 3 institutions; the fourth center did not enroll patients. The records of 10 patients were removed from the analysis because of insufficient follow-up, 6 because they were enrolled despite meeting exclusion criteria, and 7 because they underwent a second-stage surgery during the study period. The final exploratory cohort in this study comprised 100 patients who underwent a fully endoscopic surgical approach for sellar pathology. The Anterior Skull Base Nasal Inventory-12 (ASK Nasal-12) survey was completed by all 100 patients preoperatively, 99 at 2 weeks postoperatively, 95 at 3 months postoperatively, and 91 at 6 months postoperatively.

\section{Study End Points}

The prespecified primary end point of the study was patient-reported sinonasal QOL, which was determined by the ASK Nasal-12 at 2 weeks, 3 months, and 6 months after surgery. ${ }^{12}$ Secondary supplementary end points included health status estimated by patient scores on the 8-item Short-Form Health Survey (SF-8) at the same time points and sinus endoscopy findings performed at various times after surgery. ${ }^{17}$ The presence or absence and severity of mucopurulence, synechia, and crusting were recorded during nasal endoscopy.

\section{Data Collection and Analysis}

Study data, including patient demographics, surgical approach details, and patient-reported end points, were collected and entered into a research database template by a designated research nurse at each participating site. Deidentified patient information was then forwarded to the lead site at the conclusion of the study. The SF-8 responses were scored using the scoring tool provided by Quality Metric. Data were independently analyzed by a biostatistician affiliated with the lead institution.

\section{Surgical Approach and Postoperative Nasal Care}

The surgical approach for each patient was chosen by the treating neurosurgeon. Surgical details, such as treatment of the middle turbinate, sellar repair technique, and nasal packing, were tracked. To allow study of common clinical practices, there was no prescribed postoperative nasal care protocol. Nasal rinses and endoscopic debridement were prescribed at the discretion of the treating team.

\section{Statistical Analysis}

Descriptive statistics and Pearson correlations were used to assess variables as potential predictors of sinonasal QOL. Variables for the final model were selected based on a series of t-tests and backward regression models that narrowed the list of potential predictors. Potential predictors included patient demographic factors, prior surgery, tumor characteristics, surgical characteristics (e.g., implant type, graft type, and fibrin glue), and complications. Potential predictors were screened in a series of univariate exploratory analyses. The final predictors selected for the model included age, sex, use of absorbable nasal packing, nasal splints, sinusitis complication, tumor functional status, participation of an otolaryngology co-surgeon, use of nasal debridement, and self-reported SF-8 scores. Other variables of interest, such as prior surgery and history of sinusitis, were not predictive in the preliminary screening models and, therefore, were not included in the final predictive model. Potential covariates for which there were insufficient numbers to evaluate, such as nasoseptal flap, were not included in the analysis. A series of linear regressions predicting postoperative ASK Nasal-12 scores at 2 weeks, 3 months, and 6 months are presented as final models. The institution where the surgery was performed was included in our models to account for potential differences in patient self-reports by site. Scatter plots were used to visualize the association between changes in ASK Nasal-12 and SF-8 scores. The Kaplan-Meier method was used to plot symptom survival. Pairwise comparisons for symptom survival curves were conducted using log-rank and Wilcoxon tests. Values presented are 2-tailed and are considered significant at $\mathrm{p}<0.05$. SPSS version 22 was used for all analyses.

\section{Results}

In total, 100 patients who underwent fully endoscopic endonasal transsphenoidal surgery for pituitary lesions were included in this exploratory analysis. Patient charac- 
teristics are presented in Table 1 . The mean age was 51.7 \pm 15.5 years and $44 \%$ of patients were male. The majority of patients presented with nonfunctioning pituitary adenomas $(65 \%)$. Details regarding the surgical technique are presented in Table 2. A majority of patients underwent fat graft placement (54\%), co-surgery with an otolaryngologist $(55 \%)$, and postoperative nasal debridement (76\%); however, the majority of procedures did not use absorbable $(80 \%)$ or nonabsorbable $(92 \%)$ nasal packing. The middle turbinate was out-fractured rather than resected in all patients, and only 5\% had a nasoseptal flap.

Demographics and outcomes were compared by site to justify including data from multiple sites into a single analysis. Scores for ASK Nasal-12 at 2 weeks and 3 months were significantly different by site, although the effect sizes for significant comparisons were 0.03 or less, indicating a small effect. Therefore, patients were evaluated as a single cohort. The distribution of sinonasal QOL scores worsened significantly $(\mathrm{p}<0.001)$ at 2 weeks after surgery but returned to baseline by 3 months (Fig. 1). Sinonasal QOL scores remained unchanged at 6 months compared with 3 months. Overall, 57 of 95 (60\%) and 51 of $91(56 \%)$ patients had returned to baseline (preoperative) status at 3 and 6 months after surgery, respectively.

\section{Patient and Surgical Characteristics as Predictors of Sinonasal QOL}

Linear regression models were used to predict patient self-reported sinonasal QOL scores at various time points after surgery and to determine if the predictors changed during the healing process (Table 3). Potential predictors evaluated in an exploratory univariate analysis included patient demographic factors, prior surgery, tumor characteristics, surgical characteristics (e.g., implant type, graft type, and fibrin glue), and complications. Significant variables were selected for the regression models.

SF-8 scores negatively correlated with ASK-12 scores at 2 weeks $(p=0.001), 3$ months $(p<0.001)$, and 6 months $(p<0.001)$ and were the most influential predictor as evidenced by the standardized beta values shown in Table 3 . In other words, higher SF-8 scores (better health status) correlated with lower ASK Nasal-12 scores (better

\section{TABLE 1. Demographic characteristics}

\begin{tabular}{lc}
\hline \multicolumn{1}{c}{ Characteristic } & Value* \\
\hline Mean age in yrs $( \pm$ SD) & $51.7 \pm 15.5$ \\
\hline Male sex & $44(44)$ \\
\hline Tumor type & $65(65)$ \\
\hline Nonfunctioning pituitary adenoma & $9(9)$ \\
\hline Acromegaly & $7(7)$ \\
\hline Cushing's disease & $15(15)$ \\
\hline Prolactinoma & $4(4)$ \\
\hline Rathke's cleft cyst & $17.6 \pm 10.2$ \\
\hline Mean tumor size in mm ( \pm SD) & $9(9)$ \\
\hline Prior transsphenoidal surgery & $11(11)$ \\
\hline History of sinusitis & $7(7)$ \\
\hline Prior sinus surgery
\end{tabular}

* Values are number of patients (\%) unless noted otherwise.
TABLE 2. Surgical technique characteristics

\begin{tabular}{|c|c|}
\hline Characteristic & No. of Patients (\%) \\
\hline \multicolumn{2}{|l|}{ Nostril } \\
\hline Uninostril & $4(4)$ \\
\hline Binostril & $96(96)$ \\
\hline \multicolumn{2}{|l|}{ Nasal splints } \\
\hline Yes & $13(13)$ \\
\hline No & $87(87)$ \\
\hline \multicolumn{2}{|l|}{ Nasal septal flap } \\
\hline Yes & $5(5)$ \\
\hline No & $95(95)$ \\
\hline \multicolumn{2}{|l|}{ Fat graft } \\
\hline Yes & $54(54)$ \\
\hline No & $46(46)$ \\
\hline \multicolumn{2}{|l|}{ Fibrin glue } \\
\hline Yes & $45(45)$ \\
\hline No & $55(55)$ \\
\hline \multicolumn{2}{|l|}{ Dural allograft } \\
\hline Yes & $37(37)$ \\
\hline No & $63(93)$ \\
\hline \multicolumn{2}{|l|}{ Sellar implant } \\
\hline Absorbable plate & $6(6)$ \\
\hline Medpore & $36(36)$ \\
\hline Titanium & $3(3)$ \\
\hline Allograft & $1(1)$ \\
\hline Autograft & $10(10)$ \\
\hline None & $44(44)$ \\
\hline \multicolumn{2}{|c|}{ Nonabsorbable nasal packing } \\
\hline Yes & $8(8)$ \\
\hline No & $92(92)$ \\
\hline \multicolumn{2}{|c|}{ Absorbable nasal packing } \\
\hline Yes & $20(20)$ \\
\hline No & $80(80)$ \\
\hline \multicolumn{2}{|l|}{ ENT co-surgeon } \\
\hline Yes & $55(55)$ \\
\hline No & $45(45)$ \\
\hline \multicolumn{2}{|c|}{ Postop nasal debridement } \\
\hline Yes & $76(76)$ \\
\hline No & $24(24)$ \\
\hline \multicolumn{2}{|l|}{ Middle turbinate } \\
\hline Out-fracture & $100(100)$ \\
\hline Resect & $0(0)$ \\
\hline
\end{tabular}

ENT = ear, nose, and throat.

sinonasal QOL). At 2 weeks postoperatively, nasal splints emerged as a significant negative predictor $(\mathrm{p}=0.04)$, while sex was significant at the trend level $(p=0.06)$. ASK Nasal-12 scores in male patients were on average 0.36 (95\% CI -0.74 to 0.02 ) points lower (i.e., less severe symptoms) than scores in female patients at 2 weeks postoperatively. The use of nasal splints increased ASK-12 scores by an average of 0.57 points (95\% CI 0.03-1.12).

At 3 months postoperatively, the use of absorbable na- 


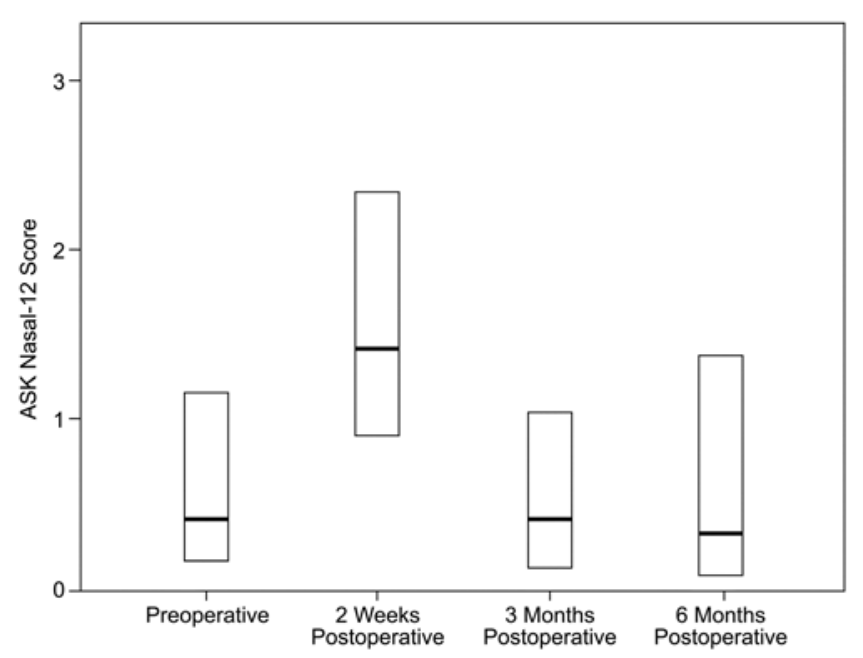

FIG. 1. Patient-recorded ASK Nasal-12 scores before surgery and at various time points after surgery. Higher scores for sinonasal QOL indicate worse symptoms. The middle line inside each box represents the median. Upper and lower horizontal lines on each box represent the 75th and 25th percentiles, respectively.

sal packing and the development of sinusitis emerged as negative predictors of sinonasal QOL $(\mathrm{p}=0.01$ and $\mathrm{p}=$ 0.03 , respectively). The use of absorbable nasal packing increased the average ASK Nasal-12 score by 0.47 points (95\% CI 0.10-0.85). Patients experiencing sinusitis as a complication reported an average ASK Nasal-12 score of 0.54 points $(95 \%$ CI $0.07-1.01)$ higher than those without sinusitis.

Interestingly, beyond the SF-8 score, none of the surgical or patient characteristics were predictive of sinonasal QOL at 6 months, likely because most patients had healed by this time. This is a reassuring result that adds credibility to the model.

\section{Relationship of Sinonasal QOL and Health Status}

In the multivariate modeling of sinonasal QOL described above, we discovered a correlation between sinonasal QOL and general measures of health status. To explore this relationship in more detail, we created 2 change scores for each measure: a difference score from the preoperative measurement to 2 weeks postoperatively and from 2 weeks postoperatively to 6 months postoperatively. Change scores between ASK Nasal-12 and SF-8 were significantly correlated with a medium effect size at both time points, that is, $r(99)=-0.32, p=0.001$ for preoperatively to 2 weeks postoperatively and $\mathrm{r}(88)=-0.38, \mathrm{p}<0.001$ for 2 weeks to 6 months postoperatively.

Scatter plots were also used to visualize the change in the ASK Nasal-12 and SF-8 scores. A positive change score for the ASK Nasal-12 and a negative change score for the SF-8 indicate that symptoms worsened (Fig. 2). At 2 weeks postoperatively, $85 \%$ of patients reported worsened sinonasal symptoms and $62 \%$ reported worsened health status; $53 \%$ of patients reported worsening on both measures. At 6 months postoperatively, 83\% of patients reported improvement in sinonasal symptoms and $76 \%$ reported improvement in health status; $69 \%$ of patients reported improvement in both measures. These data suggest that sinonasal QOL has an important association with general measures of QOL after surgery and that sinonasal QOL may influence health status during recovery.

\section{Analysis of Postoperative Nasal Endoscopy Findings}

A Kaplan-Meier analysis was performed to study time to resolution of nasal crusting, mucopurulence, and synechia as observed during nasal endoscopy after surgery (Fig. 3). The mean time to absence of nasal crusting was $16.3 \pm 2.1$ weeks $( \pm$ SEM $)$. The mean time to absence of mucopurulence was $6.2 \pm 1.1$ weeks, and the mean time to absence of synechia was $4.4 \pm 0.5$ weeks. Crusting took significantly longer than synechia $(\mathrm{p}<0.001)$ and mucopurulence $(\mathrm{p}<0.001)$ to resolve.

Multivariate models were then developed to understand the surgical techniques that may be contributing to these findings. The use of absorbable nasal packing was associated at the trend level with more severe mucopurulence $(p=0.06)$, and the use of fibrin glue was associated at the trend level with less nasal crusting $(p=0.07)$. Patients with absorbable nasal packing were 10.5 times more likely to experience moderate to severe mucopurulence, and patients with fibrin glue were 1.3 times less likely to experience moderate to severe crusting, compared with patients who did not have these treatments.

TABLE 3. Patient and surgical characteristics predicting sinonasal QOL after endoscopic transsphenoidal surgery*

\begin{tabular}{|c|c|c|c|c|c|c|c|c|c|c|c|c|}
\hline \multirow[b]{2}{*}{ Variable } & \multicolumn{4}{|c|}{2 Wks } & \multicolumn{4}{|c|}{3 Mos } & \multicolumn{4}{|c|}{6 Mos } \\
\hline & $B$ & SE B & $\beta$ & $p$ Value & $B$ & SE B & $\beta$ & $p$ Value & $B$ & SE B & $\beta$ & $p$ Value \\
\hline Age & -0.005 & 0.007 & -0.077 & 0.500 & 0.012 & 0.006 & 0.224 & 0.044 & 0.005 & 0.007 & 0.085 & 0.467 \\
\hline Female sex & -0.359 & 0.189 & -0.182 & 0.061 & -0.032 & 0.157 & -0.020 & 0.840 & -0.047 & 0.191 & -0.026 & 0.806 \\
\hline Absorbable nasal packing & 0.111 & 0.241 & 0.045 & 0.646 & 0.472 & 0.188 & 0.240 & 0.014 & 0.261 & 0.235 & 0.115 & 0.269 \\
\hline Nasal splints & 0.574 & 0.274 & 0.198 & 0.039 & -0.169 & 0.220 & -0.073 & 0.444 & -0.173 & 0.269 & -0.065 & 0.521 \\
\hline Sinusitis & -0.332 & 0.288 & -0.111 & 0.252 & 0.539 & 0.236 & 0.215 & 0.025 & 0.074 & 0.282 & 0.027 & 0.795 \\
\hline Functional tumor & -0.334 & 0.229 & -0.157 & 0.148 & -0.017 & 0.185 & -0.010 & 0.926 & -0.279 & 0.226 & -0.136 & 0.220 \\
\hline ENT co-surgeon & -0.122 & 0.221 & -0.062 & 0.583 & -0.212 & 0.185 & -0.131 & 0.255 & -0.311 & 0.226 & -0.168 & 0.173 \\
\hline Nasal debridement(s) & 0.042 & 0.252 & 0.018 & 0.869 & 0.117 & 0.203 & 0.063 & 0.567 & 0.199 & 0.253 & 0.093 & 0.434 \\
\hline SF-8 & -0.033 & 0.009 & -0.342 & 0.001 & -0.028 & 0.007 & -0.373 & $<0.001$ & -0.037 & 0.008 & -0.446 & $<0.001$ \\
\hline
\end{tabular}

$\mathrm{B}=$ beta coefficient; $\beta=$ standardized beta coefficient; $\mathrm{SE} B=$ standard error for $\mathrm{B}$.

* Significant $p$ values are those in boldface. The adjusted $r^{2}$ values were $0.19,0.25$, and 0.18 for the data obtained at 2 weeks, 3 months, and 6 months, respectively. 

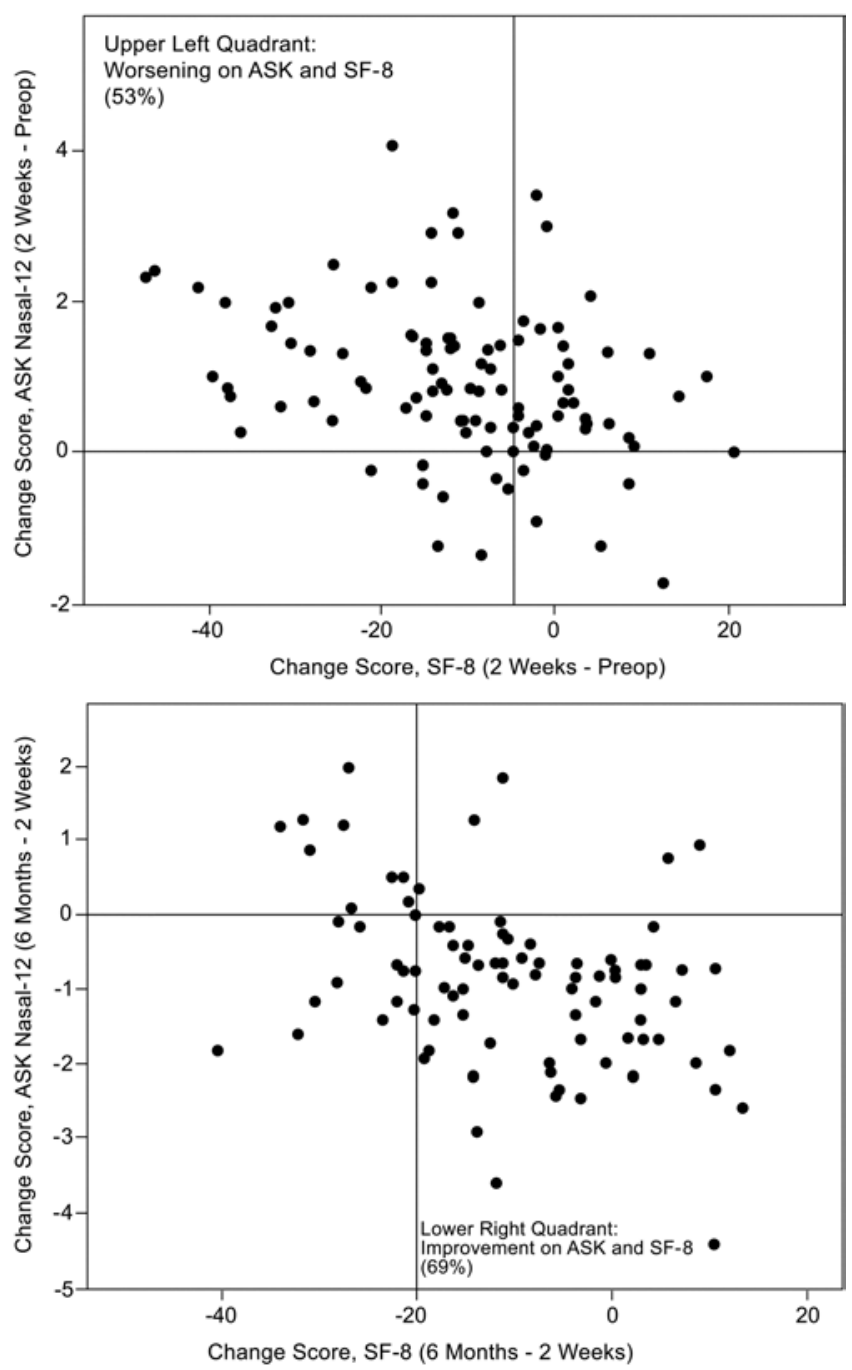

FIG. 2. Scatter plots of ASK Nasal-12 and SF-8 change scores from baseline to 2 weeks postoperatively (upper) and 2 weeks to 6 months postoperatively (lower), demonstrating the correlation between sinonasal QOL and health status.

We examined the relationship between mucopurulence and nasal packing in more detail based on the trend described above. A Kaplan-Meier analysis was performed to predict time to mucopurulence resolution for patients with and without absorbable nasal packing (Fig. 4). Patients with absorbable nasal packing had a significant increase in time to mucopurulence resolution ( $\mathrm{p}=0.046$, Wilcoxon test). Of note is the difference in the median time to symptom resolution of 6 weeks for the patients with absorbable packing versus 2 weeks for patients without.

\section{Discussion}

The goals of this study were to examine the predictors of sinonasal QOL and nasal morbidity after fully endoscopic transsphenoidal surgery to identify opportunities to improve surgical technique and improve patient counseling. We performed an exploratory post hoc analysis of a prospective cohort of 100 patients who had undergone fully endoscopic transsphenoidal surgery for pituitary le-

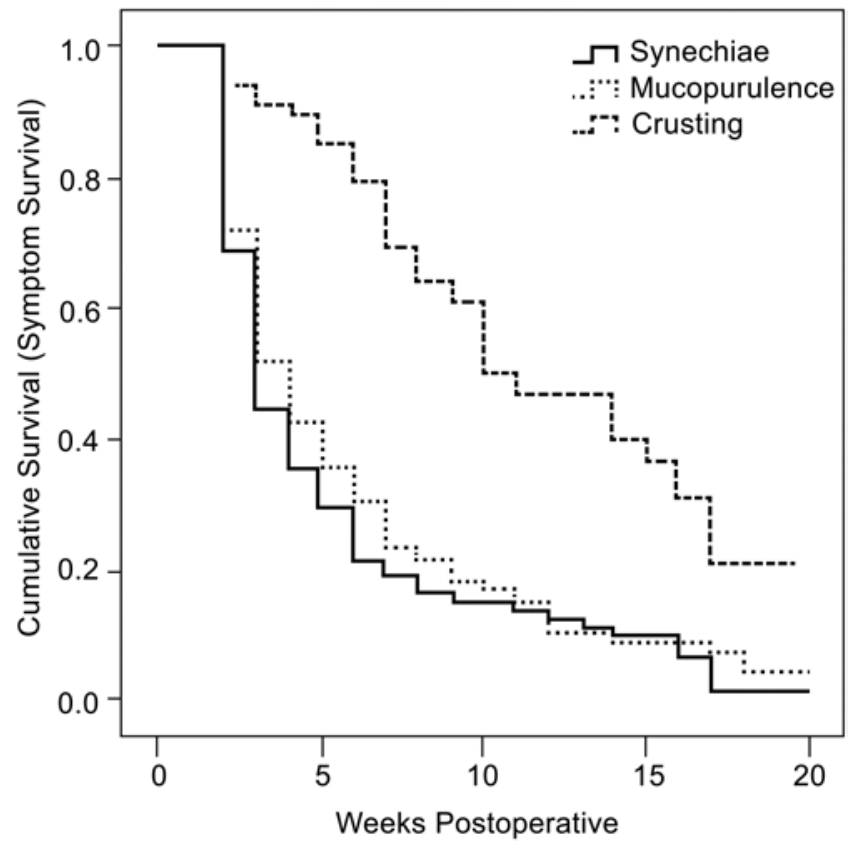

FIG. 3. Kaplan-Meier survival analysis of time to resolution of mucopurulence, nasal crusting, and synechia as observed on outpatient followup nasal endoscopy.

sions. The analysis of patient-reported outcome measures was supplemented with an analysis of objective nasal endoscopy findings.

This study has several primary findings. First, as expected, patients had significant worsening of sinonasal QOL 2 weeks after surgery, but generally improved to baseline by 3 months. This trend is now well documented with validated outcomes measures and demonstrates the time course of nasal cavity healing., ${ }^{2,15,19}$ Symptoms such as nasal congestion and nasal discharge, while troubling to patients, were temporary. Second, we learned that certain intraoperative maneuvers that impact the nasal airway, such as use of absorbable nasal packing and nasal splints used in a minority of patients, were negatively correlated with sinonasal QOL early in the postoperative period ( 2 weeks to 3 months). The development of sinusitis was also a negative predictor of early sinonasal QOL; this is an expected finding and provides internal validation of our model. However, by late follow-up (6 months), none of the intraoperative maneuvers we evaluated, including fat graft, fibrin glue, nasal packing, and otolaryngology cosurgeon, were associated with sinonasal QOL. This is reassuring because it indicates the robust capacity of the sinonasal cavity to heal after transsphenoidal surgery. Nasal splints are used to prevent synechia and long-term nasal complications, but they also seem to temporarily impact nasal QOL while they are in place. Third, the strongest association we discovered was the association between sinonasal QOL and general health status. This intriguing result illustrates the impact of sinonasal QOL on a patient's overall QOL after surgery. It reinforces the notion that sinonasal morbidity is a primary source of postoperative morbidity in these patients. Strategies designed to improve sinonasal QOL, such as reducing the use of absorbable na- 


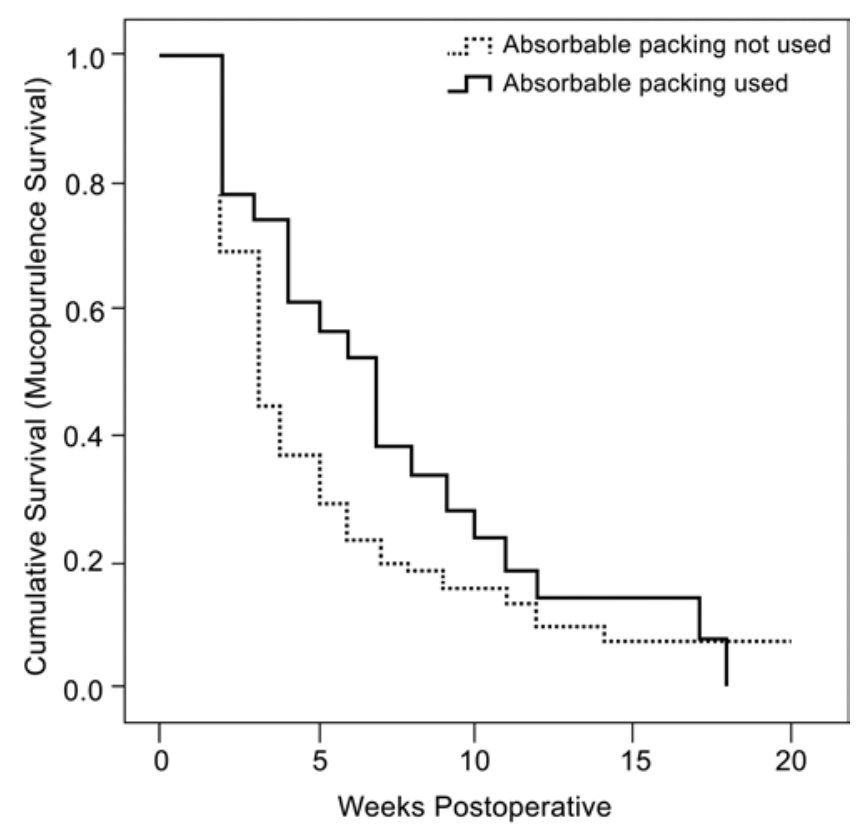

FIG. 4. Kaplan-Meier survival analysis demonstrating time to resolution of mucopurulence in patients with and without absorbable nasal packing $(p=0.046$, Wilcoxon test).

sal packing or those focused on reducing sinusitis, may improve the overall patient experience.

We also investigated the dynamics of synechia, mucopurulence, and nasal crusting resolution as objective correlates to the patient-reported measures. These findings were documented on postoperative nasal endoscopy performed in the otolaryngologist's office. Postoperative nasal debridement performed with an endoscope under local anesthesia is commonly used to clear the nasal airway and inspect healing after transsphenoidal surgery. Of these findings, crusting took the longest to resolve, followed by mucopurulence and synechia. When we examined the factors associated with these objective findings, we noted that absorbable nasal packing was associated with a higher incidence of postoperative mucopurulence and longer time to resolution. This provides an explanation for the impact of nasal packing on sinonasal QOL scores that we noted above. Absorbable nasal packing, such as NasoPore (Stryker), was used to achieve hemostasis, act as a buttress for the sellar repair, and prevent synechia formation. These data suggest that the use of absorbable nasal packing should be limited as it negatively, though temporarily, impacts sinonasal QOL. With respect to nasal crusting, we learned that fibrin glue was a positive prognostic factor at the trend level. This is an intriguing finding that needs to be validated in follow-up studies. We speculate that fibrin glue applied in the sphenoid sinus may prevent crusting by covering raw bony surfaces. Another interesting question that this work raises that would be a useful future direction is if the timing and frequency of debridement impact resolution of synechia, mucopurulence, and nasal crusting.

Interestingly, the model did not demonstrate a relationship between sinonasal QOL and otolaryngology cosurgeon during the index procedure. We would have expected patients in whom the otolaryngology team rather than the neurosurgery team performed the nasal stage of the procedure to have reported better QOL. There are several possible explanations for this. First, in one institution, the neurosurgery team performed the nasal stage except in cases that were preoperatively felt to be more difficult or in patients who had a history of sinus concerns. Patients with persistent nasal symptoms after surgery were then subsequently referred to otolaryngology for inspection. This selection may bias the results because the more complicated cases and more symptomatic patients were cared for by the otolaryngology team. Alternatively, the results support the notion that the nasal cavity has a robust capacity to heal, such that minor differences in surgical technique are undetectable by standard QOL instruments. In any case, we feel that this question deserves further analysis. These data should not, however, be used to question the important role of our otolaryngology colleagues in the endoscopic surgery technique, particularly in symptomatic patients.

There has been recent interest in QOL after skull base surgery to supplement and illuminate objective outcome measures. $2,3,6,13,16,19$ These results are providing practitioners with valuable information for managing patient expectations and modifying surgical technique to optimize QOL. A summary of recent findings is reported in Table 4.

In a univariate analysis of a mixed endoscopic skull base series, McCoul et al. identified revision surgery, extended endoscopic approaches, and use of allograft as negative prognostic factors..$^{13}$ As in our study, the authors did not detect a relationship between functioning pituitary tumors and sinonasal QOL. A multivariate analysis would have strengthened their analysis to identify the truly independent covariates. Our study did not examine the impact of extent of tumor resection on sinonasal QOL, although McCoul et al. reported subtotal resection as a negative prognostic factor. ${ }^{13}$ Pant et al..$^{15}$ studied 51 patients and de Almeida et al. ${ }^{6}$ studied 63 patients from the same institution and noted that the use of an extended approach was a negative prognostic factor. ${ }^{15}$ These studies have methodological limitations that impact the authors' conclusions. For example, the study by Pant et al. presented limited statistical analyses. ${ }^{15}$ Furthermore, all of the aforementioned studies are mixed series with respect to endonasal approaches, which limit their statistical power.

Several studies have demonstrated that different QOL domains and symptoms have variable responses after surgery. For example, Balaker et al. noted that postsurgical nasal discharge persisted longer than other physical nasal symptoms. ${ }^{2}$ Zimmer et al. noted that emotional well being was markedly improved at 3 months compared with baseline, whereas nasal physiology changes had returned to baseline by 3 months. ${ }^{19}$ Bedrosian et al. also described a dissociation of symptoms after endoscopic skull base surgery. ${ }^{3}$ They noted that while nasal symptoms tend to worsen initially after surgery, visual symptoms tend to improve.

Our study has important strengths and limitations. The strengths of the current study are its multicenter design, larger sample size than that of previous studies, inclusion of patients with only sellar pathology undergoing standard transsphenoidal approach to allow for more specific comparisons, and multivariate data modeling. Furthermore, 
TABLE 4. Summary of the predictors of sinonasal QOL after endoscopic skull base surgery

\begin{tabular}{lll}
\hline \multicolumn{1}{c}{ Predictor } & Prognostic Significance & \multicolumn{1}{c}{ Study } \\
\hline Patient characteristics & Negative & Current study (trend level) \\
\hline Female sex & Negative & Current study \\
\hline Advancing age & Positive & Current study \\
\hline Improved health status & None & Current study; McCoul et al., 2012 \\
\hline Functioning adenoma & & \\
\hline Reconstruction & Negative/none & McCoul et al., 2012; de Almeida et al., 2011 \\
\hline Septal flap & Positive & Current study; de Almeida et al., 2011 \\
\hline Fat graft/autograft & None & Current study \\
\hline Sellar implant & Negative & Current study \\
\hline Absorbable nasal packing & Negative & Current study \\
\hline Nasal splints & & \\
\hline Surgical approach & Negative & Alobid et al., 2013; de Almeida et al., 2011 \\
\hline Extended approach & Negative & McCoul et al., 2012 \\
\hline Revision surgery & None & Little et al., 2015 \\
\hline Microscopic & Positive & McCoul et al., 2012 \\
\hline Gross-total resection & & \\
\hline Complications & Negative & Current study \\
\hline Sinusitis & & Current study, Zimmer et al., 2014; McCoul et al., 2012; Balaker et al., \\
\hline Time from surgery & Positive & 2010; de Almeida et al., 2011 \\
\hline Increased duration & &
\end{tabular}

the patient-reported QOL end points are complemented by including general measures of health status and objective nasal endoscopy findings to give a more complete picture of the patient experience. One weakness of the study is that it is a post hoc analysis of a prospective cohort, which allows us to develop an association between variables but not necessarily determine causation. Furthermore, because of the experimental design, we are unable to study the impact of postoperative nasal care regimen (e.g., nasal rinses and Neti-pot) on sinonasal QOL.

Two additional points deserve discussion. The first is the variety of differences in surgical technique that were observed, such as the variable use of allografts, sellar implants, septal flap, and nasal packing. On the one hand, these variations represent a strength of the study because they allow us to evaluate the influence of these technical differences on QOL using a regression model. On the other hand, the variations limit the power of the study. The second point is the tumor population included in the study. We restricted enrollment to patients with sellar pathology that could be addressed through a transsphenoidal approach and excluded patients who had a planned extended approach. This helps limit the potential confounding influence of other skull base pathologies and extended approaches and is a strength compared with prior studies. Nevertheless, it does not eliminate the potential confounding effect the various tumor types might have on sinonasal QOL.

Questions of interest to pituitary surgeons that we did not examine are the impact of disease remission in patients with functioning pituitary tumors and the influence of postoperative endocrinopathies on sinonasal QOL. Our study is underpowered with respect to functioning pitu- itary tumors and the length of follow-up (i.e., 6 months); while this length of follow-up is sufficient for tracking sinonasal morbidity, it is not sufficient for assessing patients with functioning tumors, which can take years to evolve. For example, patients with Cushing's disease demonstrate persistent challenges in health-related QOL. ${ }^{10} \mathrm{We}$ did compare sinonasal QOL in patients with functioning tumors to that in patients with nonfunctioning tumors and did not note a significant difference. Furthermore, in the multivariate model, the presence of functioning tumor was not a predictor of sinonasal QOL (Table 3). Taken together, these observations suggest that endocrine status has limited impact on sinonasal recovery in the first 6 months after endoscopic surgery.

\section{Conclusions}

In this cohort of patients undergoing endoscopic pituitary surgery, sinonasal QOL and nasal morbidity reached a nadir at 2 weeks postsurgery and then improved significantly to baseline status in the majority of patients at 3 and 6 months postsurgery. Multivariate analysis showed that use of absorbable packing and nasal splints, older age, and female sex negatively correlate with sinonasal QOL. Sinonasal QOL and overall health status are also correlated in the postoperative period, suggesting the important influence of sinonasal QOL on the patient experience.

\section{Acknowledgments}

We would like to acknowledge the Barrow Neurological Foundation for providing funding for the study and the Neuroscience Publications office at Barrow Neurological Institute for assistance with manuscript preparation. 


\section{References}

1. Alobid I, Enseñat J, Mariño-Sánchez F, de Notaris M, Centellas S, Mullol J, et al: Impairment of olfaction and mucociliary clearance after expanded endonasal approach using vascularized septal flap reconstruction for skull base tumors. Neurosurgery 72:540-546, 2013

2. Balaker AE, Bergsneider M, Martin NA, Wang MB: Evolution of sinonasal symptoms following endoscopic anterior skull base surgery. Skull Base 20:245-251, 2010

3. Bedrosian JC, McCoul ED, Raithatha R, Akselrod OA, Anand VK, Schwartz TH: A prospective study of postoperative symptoms in sinonasal quality-of-life following endoscopic skull-base surgery: dissociations based on specific symptoms. Int Forum Allergy Rhinol 3:664-669, 2013

4. Cappabianca P, Cavallo LM, Colao A, de Divitiis E: Surgical complications associated with the endoscopic endonasal transsphenoidal approach for pituitary adenomas. J Neurosurg 97:293-298, 2002

5. Charalampaki P, Ayyad A, Kockro RA, Perneczky A: Surgical complications after endoscopic transsphenoidal pituitary surgery. J Clin Neurosci 16:786-789, 2009

6. de Almeida JR, Snyderman CH, Gardner PA, Carrau RL, Vescan AD: Nasal morbidity following endoscopic skull base surgery: a prospective cohort study. Head Neck 33:547-551, 2011

7. Dehdashti AR, Ganna A, Karabatsou K, Gentili F: Pure endoscopic endonasal approach for pituitary adenomas: early surgical results in 200 patients and comparison with previous microsurgical series. Neurosurgery 62:1006-1017, 2008

8. Hart CK, Theodosopoulos PV, Zimmer LA: Olfactory changes after endoscopic pituitary tumor resection. Otolaryngol Head Neck Surg 142:95-97, 2010

9. Jho HD, Alfieri A: Endoscopic endonasal pituitary surgery: evolution of surgical technique and equipment in 150 operations. Minim Invasive Neurosurg 44:1-12, 2001

10. Lindsay JR, Nansel T, Baid S, Gumowski J, Nieman LK: Long-term impaired quality of life in Cushing's syndrome despite initial improvement after surgical remission. J Clin Endocrinol Metab 91:447-453, 2006

11. Little AS, Kelly D, Milligan J, Griffiths C, Prevedello DM, Carrau R, et al: Comparison of sinonasal quality of life and health status in patients undergoing microscopic and endoscopic transsphenoidal surgery for pituitary lesions: a prospective cohort study. J Neurosurg [in press], 2015

12. Little AS, Kelly D, Milligan J, Griffiths C, Rosseau G, Prevedello DM, et al: Prospective validation of a patient-reported nasal quality-of-life tool for endonasal skull base surgery: The Anterior Skull Base Nasal Inventory-12. J Neurosurg 119:1068-1074, 2013

13. McCoul ED, Anand VK, Bedrosian JC, Schwartz TH: Endoscopic skull base surgery and its impact on sinonasal-related quality of life. Int Forum Allergy Rhinol 2:174-181, 2012

14. McLaughlin N, Eisenberg AA, Cohan P, Chaloner CB, Kelly DF: Value of endoscopy for maximizing tumor removal in endonasal transsphenoidal pituitary adenoma surgery. J Neurosurg 118:613-620, 2013

15. Pant H, Bhatki AM, Snyderman CH, Vescan AD, Carrau RL, Gardner P, et al: Quality of life following endonasal skull base surgery. Skull Base 20:35-40, 2010

16. Suberman TA, Zanation AM, Ewend MG, Senior BA, Ebert CS Jr: Sinonasal quality-of-life before and after endoscopic, endonasal, minimally invasive pituitary surgery. Int Forum Allergy Rhinol 1:161-166, 2011

17. Turner-Bowker DM, Bayliss MS, Ware JE Jr, Kosinski M: Usefulness of the SF-8 Health Survey for comparing the impact of migraine and other conditions. Qual Life Res 12:1003-1012, 2003

18. Zada G, Kelly DF, Cohan P, Wang C, Swerdloff R: Endonasal transsphenoidal approach for pituitary adenomas and other sellar lesions: an assessment of efficacy, safety, and patient impressions. J Neurosurg 98:350-358, 2003

19. Zimmer LA, Shah O, Theodosopoulos PV: Short-term quality-of-life changes after endoscopic pituitary surgery rated with SNOT-22. J Neurol Surg B Skull Base 75:288-292, 2014

\section{Author Contributions}

Conception and design: Little, Kelly, White. Acquisition of data: Otto, Jahnke, Chaloner, Jelinek. Analysis and interpretation of data: Little, Griffiths, Prevedello, Carrau, Chapple. Drafting the article: Little. Critically revising the article: Little, Kelly, Barkhoudarian, Otto. Reviewed submitted version of manuscript: Little, Kelly, Prevedello, Barkhoudarian. Statistical analysis: Chapple. Study supervision: Little, Kelly, Milligan, Prevedello, Rosseau.

\section{Correspondence}

Andrew S. Little, c/o Neuroscience Publications, Barrow Neurological Institute, St. Joseph's Hospital and Medical Center, 350 W. Thomas Rd., Phoenix, AZ 85013. email: neuropub@dignity health.org. 